\title{
Acute kidney injury adversely affects the clinical course of acute myeloid leukemia patients undergoing induction chemotherapy
}

\author{
Olivier Ballo ${ }^{1}$ (D) - Fagr Eladly ${ }^{1} \cdot$ Stefan Büttner ${ }^{2} \cdot$ Jan Alexander Stratmann ${ }^{1} \cdot$ Sarah Rudolf $^{2} \cdot$ Uta Brunnberg $^{1}$. \\ Eva-Maria Kreisel $^{1} \cdot$ Björn Steffen ${ }^{1,3} \cdot$ Sebastian Wagner ${ }^{1,3} \cdot$ Fabian Finkelmeier $^{4} \cdot$ Hubert Serve $^{1,3}$. \\ Christian H. Brandts ${ }^{1,3,5}$
}

Received: 25 December 2020 / Accepted: 2 March 2021 / Published online: 11 March 2021

(C) The Author(s) 2021

\begin{abstract}
Acute kidney injury (AKI) complicates the clinical course of hospitalized patients by increasing need for intensive care treatment and mortality. There is only little data about its impact on AML patients undergoing intensive induction chemotherapy. In this study, we analyzed the incidence as well as risk factors for AKI development and its impact on the clinical course of AML patients undergoing induction chemotherapy. We retrospectively analyzed data from 401 AML patients undergoing induction chemotherapy between 2007 and 2019. AKI was defined and stratified according to KIDGO criteria by referring to a defined baseline serum creatinine measured on day 1 of induction chemotherapy. Seventy-two of 401 (18\%) AML patients suffered from AKI during induction chemotherapy. AML patients with AKI had more days with fever (7 vs. 5, $p=0.028)$ and were more often treated on intensive care unit $(45.8 \%$ vs. $10.6 \%, p<0.001)$. AML patients with AKI had a significantly lower complete remission rate after induction chemotherapy and, with 402 days, a significantly shorter median overall survival (OS) (median OS for AML patients without AKI not reached). In this study, we demonstrate that the KIDGO classification allows mortality risk stratification for AML patients undergoing induction chemotherapy. Relatively mild AKI episodes have impact on the clinical course of these patients and can lead to chronic impairment of kidney function. Therefore, we recommend incorporating risk factors for AKI in decision-making considering nutrition, fluid management, as well as the choice of potentially nephrotoxic medication in order to decrease the incidence of AKI.
\end{abstract}

Keywords Acute kidney failure $\cdot$ Acute myeloid leukemia $\cdot$ Induction chemotherapy $\cdot$ Survival $\cdot$ Intensive care treatment

Olivier Ballo and Fagr Eladly contributed equally to this work

Olivier Ballo

olivier.ballo@kgu.de

$\triangle$ Christian H. Brandts

christian.brandts@kgu.de

1 Department of Medicine, Hematology/Oncology, University Hospital, Goethe University, Frankfurt, Germany

2 Department of Medicine, Nephrology, University Hospital, Goethe University, Frankfurt, Germany

3 German Cancer Consortium (DKTK) and German Cancer Research Center (DKFZ), Heidelberg, Germany

4 Department of Medicine, Gastroenterology, Hepatology and Endocrinology, University Hospital, Goethe University, Frankfurt, Germany

5 University Cancer Center Frankfurt (UCT), University Hospital, Goethe University, Frankfurt, Germany

\section{Introduction}

Acute myeloid leukemia (AML) is a hematological malignancy arising from a clonal proliferation of myeloid precursors losing their ability to differentiate into mature functional blood cells. A curative therapy approach can only be achieved by intensive induction chemotherapy. During the past 30 years, advances in intensified chemotherapy, allogenic stem cell transplantation (SCT), and supportive measures resulted in increased survival rates [1, 2]. Still, AML patients undergoing induction chemotherapy are at high risk to develop complications and treatment-related mortality (TRM) remains at a level of $8-15 \%[3,4]$.

Acute kidney injury (AKI) is defined by a rapid and partly reversible restriction of kidney function causing a reduction of renal glomerular filtration rate (GFR) [5]. AKI has been shown to be associated with increased mortality and a higher rate of treatment on intensive care units (ICU) in hospitalized 
patients $[5,6]$. In pediatric AML patients undergoing intensive chemotherapy, AKI has been correlated to volume depletion, sepsis, and nephrotoxic medications, and a higher risk for developing chronic kidney disease later [7]. Pediatric AML patients with AKI showed a significantly higher mortality rate than AML patients without AKI. Lahoti et al. revealed that $36 \%$ of adult AML patients develop AKI during chemotherapy [8]. Advanced age, mechanical ventilation, vasopressors, low WBC, hypoalbuminemia, use of vancomycin, diuretics, and liposomal amphotericin B were positively associated with the occurrence of AKI. Furthermore, the probability of achieving a complete remission (CR) was shown to be significantly lower for AML patients who developed AKI compared to those without AKI. To our knowledge, only the study by Lahoti et al. have investigated the impact of AKI in adult AML patients undergoing intensive chemotherapy.

We conducted this retrospective study to determine the incidence of AKI as defined and classified by Kidney Disease: Improving Global Outcomes (KDIGO) Clinical Practice Guideline in AML patients undergoing induction chemotherapy at our institute (https://kdigo.org/wp-content/ uploads/2016/10/KDIGO-2012-AKI-Guideline-English.pdf). Furthermore, we aimed at identifying potential risk factors for $\mathrm{AKI}$ as well as associated clinical conditions.

\section{Materials and methods}

\section{Study design and treatment protocols}

In this single-center study, we retrospectively included all patients aged $\geq 18$ with AML (excluding acute promyelocytic leukemia) who underwent intensive induction chemotherapy between 2007 and 2019. AKI was defined and stratified as recommended by the KDIGO Clinical Practice Guideline (https://kdigo.org/wp-content/uploads/2016/10/KDIGO2012-AKI-Guideline-English.pdf). AML patients with an increase in serum creatinine ( $\mathrm{SCr}$ ) by $\geq 0.3 \mathrm{mg} / \mathrm{dl}$ within $48 \mathrm{~h}$ or an increase in $\mathrm{SCr}$ to $\geq 1.5$ times baseline within the prior 7 days during the hospital stay of induction chemotherapy were defined as AML patients with AKI. SCr increase 1 . 5-1.9 times baseline or $\geq 0.3 \mathrm{mg} / \mathrm{dl}$ was defined as AKI stage 1, and SCr 2.0-2.9 times baseline was defined as AKI stage 2 and $\mathrm{SCr} 3.0$ times baseline or increase in serum creatinine to $\geq$ $4.0 \mathrm{mg} / \mathrm{dl}$ or initiation of renal replacement therapy was defined as AKI stage 3 (Table S1). All other patients were defined as AML patients without AKI. The baseline SCr was defined as the value measured at day 1 of induction chemotherapy. AML patients with a baseline $\mathrm{SCr} \geq 1.5 \mathrm{mg} / \mathrm{dl}$ were excluded from this study. Urine output was not considered for definition and stratification of AKI. Standard induction chemotherapy was the so-called 7+3-regime; cytarabine $100 \mathrm{mg} /$ $\mathrm{m}^{2}$ given intravenous (IV) continuously for 7 days is combined with daunorubicin $60 \mathrm{mg} / \mathrm{m}^{2}$ given as a $30-\mathrm{min}$ IV infusion on days 3,4 , and 5 [9]. Patients under the age of 60 received a second induction therapy with $7+3$ if early blast clearance was achieved in $\mathrm{d} 15$ bone marrow blood evaluation or with HAM protocol (cytarabine $3000 \mathrm{mg} / \mathrm{m}^{2}$ was administered by 3 -h IV infusion every $12 \mathrm{~h}$ on day 1 through 3 and mitoxantrone $10 \mathrm{mg} / \mathrm{m}^{2}$ by 30 -min IV infusion on days 3,4 , and 5) if blast clearance was not achieved on d15 bone marrow blood evaluation [10]. Patients above the age of 60 received only a second induction chemotherapy with HAM (with reduced cytarabine dose of $1000 \mathrm{mg} / \mathrm{m}^{2}$ ), if the first induction therapy cycle was not sufficient to achieve bone marrow blast clearance on d15 [11]. In case of a complete remission $(\mathrm{CR})$ after induction chemotherapy with $7+3$ alone or with $7+3$ and HAM, patients went on to receive a consolidation treatment with either high-dose cytarabine or with an allogenic SCT. Response assessment was performed in accordance with the European Leukemia Net (ELN) recommendations from 2010 [12]. Patients received routinely antimicrobial prophylaxis with levofloxacin and posaconazole daily as suggested by current guidelines [13, 14]. A day with fever was defined as a body temperature increase above $\geq 38.3{ }^{\circ} \mathrm{C}$ once or $\geq 38.0^{\circ} \mathrm{C}$ on two consecutive days [15]. If fever or a significant increase of C-reactive protein (CRP) (doubling of CRP level and absolute value above $5 \mathrm{mg} / \mathrm{dl}$, norm $<0.5$ $\mathrm{mg} / \mathrm{dl}$ ) was found, antibiotic prophylaxis was replaced by intravenous broad-spectrum antibiotics. Blood testing (hematology, liver and kidney function, coagulation, inflammation markers) was performed every other day routinely.

The study was performed in accordance with the 2013 Helsinki declaration. Patients provided informed written consent to retrospective data extraction from patient charts and patient data was provided after approval by the local Ethics Committee (approval number SHN-01-2020). The ethics committee waived the requirement for informed consent for deceased patients. In addition, the majority of patients were also enrolled in the AML registry of the Study Alliance Leukemia (approval number EK 98032010). After ethics approval, patient data was retrieved from the clinical cancer registry of the University Cancer Center (UCT) Frankfurt, complemented by data directly from the medical records and fully anonymized. Data analysis was performed on anonymized data.

\section{Statistical analysis}

This study was designed as a retrospective cohort study. Patients were followed until death or last contact. Dates of treatment start and finish with induction chemotherapy were assessed separately. Continuous variables are shown as median \pm range and categorical variables are reported as frequencies and percentages. All continuous variables were tested for normality and were analyzed by using the Student's $t$-test or 
the Wilcoxon-Mann-Whitney test accordingly. Chi-squared test was used for binary variables. Death rates were analyzed by Kaplan-Meier method and curves were compared by logrank test. Predictors of survival were determined using a univariate Cox regression hazard model. Death was recorded as an event. Statistical analysis was performed with SPSS (Version 22.0, IBM, Armonk, NY, USA).

\section{Results}

Four hundred one patients diagnosed with AML between 2007 and 2019 that underwent intensive induction therapy were included in this retrospective analysis. According to the KDIGO classification, 72 (18\%) AML patients suffered from AKI during the hospital stay of induction chemotherapy and were assigned to the AML cohort with AKI, and 329 (82\%) AML patients did not suffer from AKI during induction chemotherapy and were assigned to the AML cohort without AKI. SCr increase defining AKI was evaluated with respect to a baseline $\mathrm{SCr}$ measured at day 1 of induction chemotherapy. Twenty-two AML patients with a baseline $\mathrm{SCr} \geq 1.5$ were excluded from further analysis. Urine output was not considered for definition and stratification of AKI.

\section{Baseline characteristics of AML patients with and without AKI}

Median age was 61 years (range 24-78) in AML patients with AKI and 58 years (range 18-82) in AML patients without AKI $(p=0.014)$ (Table 1). The fraction of AML patients older than 60 years of age was $51.4 \%$ in AML patients with AKI and $41.6 \%$ in AML patients without AKI $(p=0.149)$. The median albumin level on the day of admission was $3.7 \mathrm{~g} / \mathrm{dl}$ (24.9) in AML patients with AKI and $3.8 \mathrm{~g} / \mathrm{dl}(2-5.2)$ in AML patients without AKI $(p=0.015)$. There was no significant difference between both cohorts with respect to gender, WHO classification, blood counts, and other blood test results [16]. AML risk groups according to the European Leukemia Net (ELN) recommendations from 2010 were equally distributed between the two cohorts $(p=0.923)$ [12]. Thirty-one (43.1\%) of the AML patients with AKI suffered from AKI KDIGO stage 1, 30 (41.7\%) from AKI KDIGO stage 2, and 11 (15.3\%) from AKI KDIGO stage 3 (Table 2). Median days to the first AKI episode during induction chemotherapy were 18.5 days (1-120). In median, AML patients with AKI suffered from 1 AKI episode (range 1-3).

\section{Clinical findings in AML patients with and without AKI}

There was no significant difference between the two cohorts with respect to the length of the hospital stay for induction chemotherapy (46 days vs. 49 days, $p=0.237$ ) (Table 3 ).
AML patients with AKI had a median of 7 (0-23) days with fever compared to $5(0-31)$ days with fever in AML patients without AKI $(p=0.028)$. Thirty-three $(45.8 \%)$ AML patients with AKI required treatment on ICU, significantly more than AML patients without AKI $(n=35,10.6 \%, p<0.001)$. During the hospital stay of induction chemotherapy, AML patients with AKI received $12(0-53)$ RBC transfusion and 11 (273) platelet concentrates compared to $10(0-35)$ RBC transfusion and $8(0-33)$ platelet concentrates $(p=0.152$ and $p=$ 0.100 respectively). The percentage of patients receiving two cycles of induction chemotherapy was significantly lower in AML patients with AKI, $(33.3 \%$ vs. $53.8 \%, p<0.001)$. Thirty-four (47.2\%) AML patients with AKI received allogenic SCT as consolidation therapy compared to 193 (58.7\%) AML patients without AKI $(p=0.088)$. Only $39(54.2 \%)$ AML patients with AKI achieved a CR after completion of induction chemotherapy, whereas in AML patients without AKI, 254 (77.2\%) received a CR after completion of induction chemotherapy $(p=0.005)$.

\section{Laboratory findings in AML patients with and without AKI}

Median hemoglobin levels and platelet counts measured throughout the hospital stay of induction chemotherapy did not differ significantly in AML patients with and without AKI (Table 4). AML patients with AKI had lower albumin levels $(3.2 \mathrm{~g} / \mathrm{dl}$, range $2.1-4.0$, vs. $3.4 \mathrm{~g} / \mathrm{dl}$, range $2.25-4.25, p$ $<0.001$ ) and higher CRP levels during the hospital stay of induction chemotherapy than AML patients without AKI $(5.94 \mathrm{mg} / \mathrm{dl}$, range $0.61-34.66$, vs. $3.69 \mathrm{mg} / \mathrm{dl}$, range 0.19 $87.6, p<0.001)$. Median $\mathrm{SCr}$ levels during the hospital stay of induction chemotherapy were $0.83 \mathrm{mg} / \mathrm{dl}(0.45-3.03)$ in AML patients with AKI and $0.71 \mathrm{mg} / \mathrm{dl}(0.37-1.27)$ in AML patients without AKI $(p<0.001)$. SCr levels remained higher in AML patients with AKI at discharge $(1.02 \mathrm{mg} / \mathrm{dl}$, range $0.37-2.40$, vs. $0.71 \mathrm{mg} / \mathrm{dl}$, range $0.33-1.21, p<0.001)$ and on day 90 after initiation of induction chemotherapy $(0.98 \mathrm{mg} /$ $\mathrm{dl}$, range $0.37-1.72$, vs. $0.72 \mathrm{mg} / \mathrm{dl}$, range $0.2-3.42, p<$ $0.001)$.

All $p$-values reported are two-sided, and statistical significance was defined as $p \leq 0.05$

\section{Distribution of medication in AML patients with and without AKI}

The distribution of anti-infective agents used in AML patients with and without AKI is illustrated in Table 5. Sixty-one (84.7\%) AML patients with AKI had exposure to fluoroquinolones compared to 304 (92.4\%) AML patients without AKI $(p=0.065)$. Exposure to other antibiotics (acylaminopenicillin $+\beta$-lactamase inhibitor, carbapenems, and glycopeptides) did not differ between AML patients with 
Table 1 Baseline characteristics

\begin{tabular}{|c|c|c|c|c|}
\hline Characteristic & All & AML with AKI & AML without AKI & $p$-value \\
\hline Number of patients $(n, \%)$ & 401 & $72(18)$ & $329(82)$ & \\
\hline Median age (median, range) & $58(18-82)$ & $61(24-78)$ & $58(18-82)$ & 0.014 \\
\hline Patients above the age of $60(n, \%)$ & $174(43.39)$ & $37(51.4)$ & 137 (41.6) & 0.149 \\
\hline Female sex $(n, \%)$ & $188(46.88)$ & $32(44.44)$ & $156(47.42)$ & 0.697 \\
\hline Favorable ELN risk group $(n, \%)$ & $82(20.45)$ & $13(18.06)$ & $69(20.97)$ & 0.923 \\
\hline Intermediate-I ELN risk group $(n, \%)$ & $150(37.41)$ & $28(38.89)$ & $122(37.08)$ & 0.923 \\
\hline Intermediate-II ELN risk group $(n, \%)$ & $91(22.69)$ & $16(22.22)$ & $75(22.80)$ & 0.923 \\
\hline Adverse ELN risk group $(n, \%)$ & $71(17.71)$ & $11(15.28)$ & $60(18.23)$ & 0.923 \\
\hline AML with recurrent genetic abnormalities $(n, \%)$ & $170(42.39)$ & $26(36.11)$ & $144(43.79)$ & 0.425 \\
\hline AML with myelodysplasia-related changes $(n, \%)$ & $58(14.46)$ & $16(22.22)$ & $42(12.77)$ & 0.425 \\
\hline Therapy-related myeloid neoplasms $(n, \%)$ & $5(1.25)$ & $1(1.39)$ & $4(1.22)$ & 0.425 \\
\hline AML not otherwise specified $(n, \%)$ & $165(41.15)$ & $29(40.28)$ & $136(41.34)$ & 0.425 \\
\hline Albumin $\mathrm{g} / \mathrm{dl}$ (median, range)* & $3.8(2.0-5.2)$ & $3.70(2.0-4.9)$ & $3.8(2.0-5.2)$ & 0.015 \\
\hline White blood count/nl (median, range)* & $10.71(0.38-340.0)$ & $8.60(0.38-340.0)$ & $10.90(0.38-324.73)$ & 0.670 \\
\hline Hemoglobin g/dl (median, range)* & $9.10(0.35-16.20)$ & $8.95(4.5-14.6)$ & $9.10(0.35-16.20)$ & 0.469 \\
\hline Thrombocytes count $/ \mathrm{nl}$ (median, range)* & $56.00(3-836)$ & $51.00(6-590)$ & $58.00(3-836)$ & 0.375 \\
\hline Creatinine baseline $\mathrm{mg} / \mathrm{dl}$ (median, range) & $0.83(0.41-1.49)$ & $0.80(0.48-1.45)$ & $0.90(0.41-1.49)$ & 0.846 \\
\hline C-reactive protein $\mathrm{mg} / \mathrm{dl}$ (median, range)* & $2.61(0.01-43.48)$ & $2.69(0.02-42.74)$ & $2.58(0.01-43.48)$ & 0.243 \\
\hline
\end{tabular}

*At time of admission to hospital. All $p$-values reported are two-sided, and statistical significance was defined as $p \leq 0.05$

and without AKI. Sixty (83.3\%) AML patients with AKI received azole antifungals during the hospital stay, significantly less than $303(92.1 \%)$ AML patients without AKI $(p=0.027)$. Liposomal amphotericin B was more often used in AML patients with AKI than in AML patients without AKI (23.6\% vs. $10.9 \%, p=0.007)$. Exposition to angiotensin-converting enzyme (ACE) inhibitors was significantly higher in AML patients with AKI than in AML patients without AKI $(n=24$, $36.1 \%$, vs. $n=63,19.1 \%, p=0.003$ ).

All $p$-values reported are two-sided, and statistical significance was defined as $p \leq 0.05$

\section{Risk factors for AKI in AML patients undergoing induction chemotherapy}

To further analyze risk factors for AKI in AML patients undergoing induction chemotherapy, a uni- and multivariate logistic regression model was performed. The nominal dichotome variables age above 60 years, male sex, $>5$ days with fever, treatment on ICU, treatment with ACE inhibitors, and treatment with liposomal amphotericin B were included in this model. As shown in Table 6, treatment on ICU and treatment with ACE inhibitors or liposomal amphotericin B were independent risk factors for AKI in AML patients undergoing induction chemotherapy.

Risk factors AKI were determined using a uni- and multivariate binary logistic regression model. All $p$ - values reported are two-sided, and statistical significance was defined as $p \leq 0.05$

\section{Outcome}

We analyzed the survival of both patient cohorts. Overall and 90-day mortality were significantly higher in AML patients with AKI, $45(62.5 \%)$ vs. 130 (39.5\%) and $22(30.6 \%)$ vs. 11 $(3.3 \%)$ respectively ( $p=0.001$ and $<0.001$ respectively) (Table 3). For AML patients without AKI during the hospital stay of induction chemotherapy, median OS was not reached. For AML patients with AKI during the hospital stay of induction chemotherapy, median OS was 402 days $(95 \%$

Table 2 Characterization of AML patients with AKI during induction chemotherapy

\begin{tabular}{ll}
\hline Characteristic & AML with AKI \\
\hline Number of patients $(n, \%)$ & $72(18)$ \\
AKI KDIGO stage $1(n, \%)$ & $31(43.1)$ \\
AKI KDIGO stage $2(n, \%)$ & $30(41.7)$ \\
AKI KDIGO stage $3(n, \%)$ & $11(15.3)$ \\
Days to first AKI (median, range) & $18.5(1-120)$ \\
Total number of AKI (median, range) & $1(1-3)$
\end{tabular}

*Median of values recorded at day of discharge. All $p$-values reported are two-sided, and statistical significance was defined as $p \leq 0.05$ 
Table 3 Clinical findings in AML patients with and without AKI

\begin{tabular}{llll}
\hline Characteristic & AML with AKI & AML without AKI & $p$-value \\
\hline Number of patients $(n, \%)$ & $72(18)$ & $329(82)$ & \\
Length of hospital stay (median, range) & $46(8-127)$ & $49(5-125)$ & 0.237 \\
Days with fever (median, range) & $7(0-23)$ & $5(0-31)$ & 0.028 \\
Patients requiring treatment on intensive care unit $(n, \%)$ & $33(45.8)$ & $35(10.6)$ & $<0.001$ \\
Transfused RBC concentrates (median, range) & $12(0-53)$ & $10(0-35)$ & 0.152 \\
Transfused platelet concentrates (median, range) & $11(2-73)$ & $8(0-33)$ & 0.100 \\
Two cycles of induction chemotherapy $(n, \%)$ & $24(33.3)$ & $177(53.8)$ & $<0.001$ \\
Stem cell transplantation as consolidation therapy $(n, \%)$ & $34(47.2)$ & $193(58.7)$ & 0.088 \\
Complete remission after induction chemotherapy $(n, \%)$ & $39(54.2)$ & $254(77.2)$ & 0.005 \\
Overall mortality $(n, \%)$ & $45(62.5)$ & $130(39.5)$ & 0.001 \\
90-day mortality $(n, \%)$ & $22(30.6)$ & $11(3.3)$ & $<0.001$ \\
\hline
\end{tabular}

* Median of values recorded at day of discharge. All $p$-values reported are two-sided, and statistical significance was defined as $p \leq 0.05$ confidence interval 87-718). We further analyzed OS with respect to the different KDIGO stages of AKI. The median OS for AML patients with AKI KDIGO stage 1 was not reached, for AML patients with KDIGO stage 2366 days (95\% confidence interval 0-1065), and for AML patients with KDIGO stage 3220 days (95\% 0-451). The Kaplan-Meier estimates with a stepwise increase of mortality with increasing AKI severity are displayed in Fig. 1.

To further analyze AKI as a prognostic parameter in AML patients undergoing intensive induction chemotherapy, a uniand multivariate Cox regression model with forward stepwise likelihood ratio was performed. The nominal dichotome variables age above 60 years, male sex, adverse-risk AML, treatment on ICU, CR after induction chemotherapy, allogenic SCT as consolidation therapy, and AKI during induction chemotherapy were included in this model. As shown in Table 7, age above 60 years, adverse-risk AML, treatment on ICU, CR after induction chemotherapy, allogenic SCT as consolidation therapy, and AKI during induction chemotherapy were independently associated with OS. Importantly, AKI remained as an independent risk factor for OS, when censored for AKI grade 3 .
$C I$, confidence interval; $H R$, hazard ratio. All $p$-values reported are two-sided, and statistical significance was defined as $p \leq 0.05$

\section{Discussion}

During the past 30 years, advances in the management of AML patients undergoing intensified chemotherapy and bone marrow transplantation as well as improvements in supportive measures resulted in increasing survival rates [1,2]. However, TRM remains at a level of $8-15 \%$ during induction chemotherapy $[3,4]$. AKI is one of the most important organ failures associated with increased mortality and need for ICU treatment in hospitalized patients $[6,17]$. To the best of our knowledge, the last investigation on the role of AKI in adult patients with AML undergoing intensive induction therapy was published in 2010, while the most recent investigation focused on pediatric AML populations [7, 8]. In this study, we evaluated risk factors for AKI in AML patients undergoing induction chemotherapy and show that AKI adversely affects the
Table 4 Laboratory findings in AML patients with and without AKI during the hospital stay of induction chemotherapy

\begin{tabular}{llll}
\hline Characteristic & AML with AKI & AML without AKI & $p$-value \\
\hline Number of patients $(n, \%)$ & $72(18)$ & $329(82)$ & \\
Hemoglobin g/dl (median, range) & $8.9(7.40-12.00)$ & $9(6.40-13.15)$ & 0.458 \\
Platelet count/nl (median, range) & $25.5(7.0-172.0)$ & $28(5.5-210)$ & 0.100 \\
Albumin g/dl (median, range) & $3.2(2.1-4.0)$ & $3.4(2.3-4.3)$ & $<0.001$ \\
C-reactive protein mg/dl (median, range) & $5.94(0.61-34.66)$ & $3.69(0.19-87.60)$ & $<0.001$ \\
Creatinine mg/dl (median, range) & $0.83(0.45-3.03)$ & $0.71(0.37-1.27)$ & $<0.001$ \\
Creatinine at discharge mg/dl (median, range) & $1.02(0.37-2.40)$ & $0.71(0.33-1.21)$ & $<0.001$ \\
Creatinine day 90 mg/dl (median, range) & $0.98(0.37-1.72)$ & $0.72(0.20-3.42)$ & $<0.001$ \\
Highest level of procalcitonin ng/ml (median, range) & $1.80(0.09-86.00)$ & $0.40(0.04-159.20)$ & $<0.001$ \\
\hline
\end{tabular}


Table 5 Distribution of medication in AML patients with and without AKI

\begin{tabular}{llll}
\hline Characteristic & AML with AKI & AML without AKI & $p$-value \\
\hline Number of patients $(n, \%)$ & $72(18)$ & $329(82)$ & \\
Fluoroquinolone $(n, \%)$ & $61(84.7)$ & $304(92.4)$ & 0.065 \\
Acylaminopenicillin + -lactamase inhibitor $(n, \%)$ & $45(62.5)$ & $213(64.7)$ & 0.786 \\
Carbapenems $(n, \%)$ & $57(79.2)$ & $259(78.7)$ & 1.000 \\
Glycopeptides $(n, \%)$ & $56(77.8)$ & $248(75.4)$ & 0.762 \\
Azole antifungals $(n, \%)$ & $60(83.3)$ & $303(92.1)$ & 0.027 \\
Caspofungin $(n, \%)$ & $27(37.5)$ & $110(33.4)$ & 0.583 \\
Aciclovir $(n, \%)$ & $29(40.3)$ & $138(42.0)$ & 0.895 \\
Liposomal amphotericin B $(n, \%)$ & $17(23.6)$ & $36(11.0)$ & 0.007 \\
Angiotensin-converting enzyme inhibitors $(n, \%)$ & $26(36.1)$ & $63(19.1)$ & 0.003 \\
\hline
\end{tabular}

clinical course in these patients with poor CR rates after induction chemotherapy and impaired survival.

We found that AKI, defined according to the most recent KIDGO criteria, was present in $18 \%$ of AML patients undergoing induction chemotherapy. The median time to development of AKI was 18.5 days. The median time to AKI described by Lahoti et al. was also 18 days; however, the incidence of AKI was $36 \%$ significantly higher than in our study, although the RIFLE classification (used by Lahoti et al.) is more conservative than the KDIGO classification [8]. Notably, when detecting SCr increase in our study, we referred (different from Lahoti et al.) to a defined baseline $\mathrm{SCr}$ level from day 1 of induction chemotherapy. We thereby aimed to avoid detecting $\mathrm{AKI}$ by a SCr increase following on an inadequately low SCr level (e.g., due to dilution by iatrogenic fluid overload), which would be detected as AKI by Lahoti et al. but not in our study. Another explanation may be the different induction treatment protocols used in the study of Lahoti. Whereas in our study, standard induction chemotherapy was the so-called 7+3-regime; a relevant proportion of the AML population studied by Lahoti at al. received different chemotherapy regimens including the purine analogues clofarabine and fludarabine known to potentially cause AKI [18]. Finally, improvement in supportive measures in the last 10 years, e.g., by avoiding nephrotoxic drugs, is likely to contribute to the lower AKI incidence in our AML cohort.
AML patients with AKI had more days with fever than AML patients without AKI and presented with higher levels of inflammation parameters (PCT and CRP) throughout the hospital stay of induction chemotherapy. In this study, we confirmed the association of AKI and ICU treatment in AML patients as already described by others [8, 19]. Sepsisassociated inflammatory response with a potential disruption of renal perfusion as a consequence is known to be a common risk factor for AKI (https://kdigo.org/wp-content/uploads/ 2016/10/KDIGO-2012-AKI-Guideline-English.pdf) [6, 20].

$\mathrm{CR}$ is a predictor of mortality in patients with AML $[7,8]$. In our investigation, we were able to show that $\mathrm{CR}$ rates were significantly lower in AML patients with AKI. It is obvious that the significantly lower CR rate in these AML patients cannot be simply attributed to AKI itself. The percentage of AML patients receiving two cycles of induction chemotherapy was significantly lower in all AML patients with AKI and also in AML patients with AKI younger than 60 years (regularly receiving two cycles of induction chemotherapy). We speculate that CR rates in AML patients with AKI are lower, because AML patients with AKI suffer from infectious complications and treatment on ICU. Consequently, initially intended treatment cannot be pursued or may be disrupted in AML patients with AKI. This finding is supported by the fact that patients with AKI are less likely to receive allogenic SCT as consolidation therapy and are consequently less likely to

Table 6 Logistic regression analysis of risk factors for AKI

\begin{tabular}{|c|c|c|c|c|c|c|}
\hline Parameter & \multicolumn{3}{|c|}{ Univariate analysis } & \multicolumn{3}{|c|}{ Multivariate analysis } \\
\hline Age $>60$ & 1.482 & $0.888-2.471$ & 0.132 & & & \\
\hline Male sex & 1.127 & $0.675-1.882$ & 0.647 & & & \\
\hline$>5$ days with fever & 1.552 & $0.919-2.618$ & 0.100 & & & \\
\hline Treatment on intensive care unit & 7.108 & $3.975-12.709$ & $<0.001$ & 7.885 & $4.250-14.628$ & $<0.001$ \\
\hline Treatment with angiotensin-converting enzyme inhibitors & 2.386 & $1.372-4.152$ & 0.002 & 2.931 & $1.574-5.457$ & 0.001 \\
\hline Treatment with liposomal amphotericin B & 2.516 & $1.320-4.793$ & 0.005 & 2.186 & $1.055-4.529$ & 0.035 \\
\hline
\end{tabular}


Fig. 1 Kaplan-Meier curves for overall survival (OS). OS of AML patients without AKI (solid line) and AML patients with KDIGO stage 1 AKI (dashed line), KDIGO stage 2 AKI (dotted line), and KDIGO stage 3 AKI (dotted and dashed line)

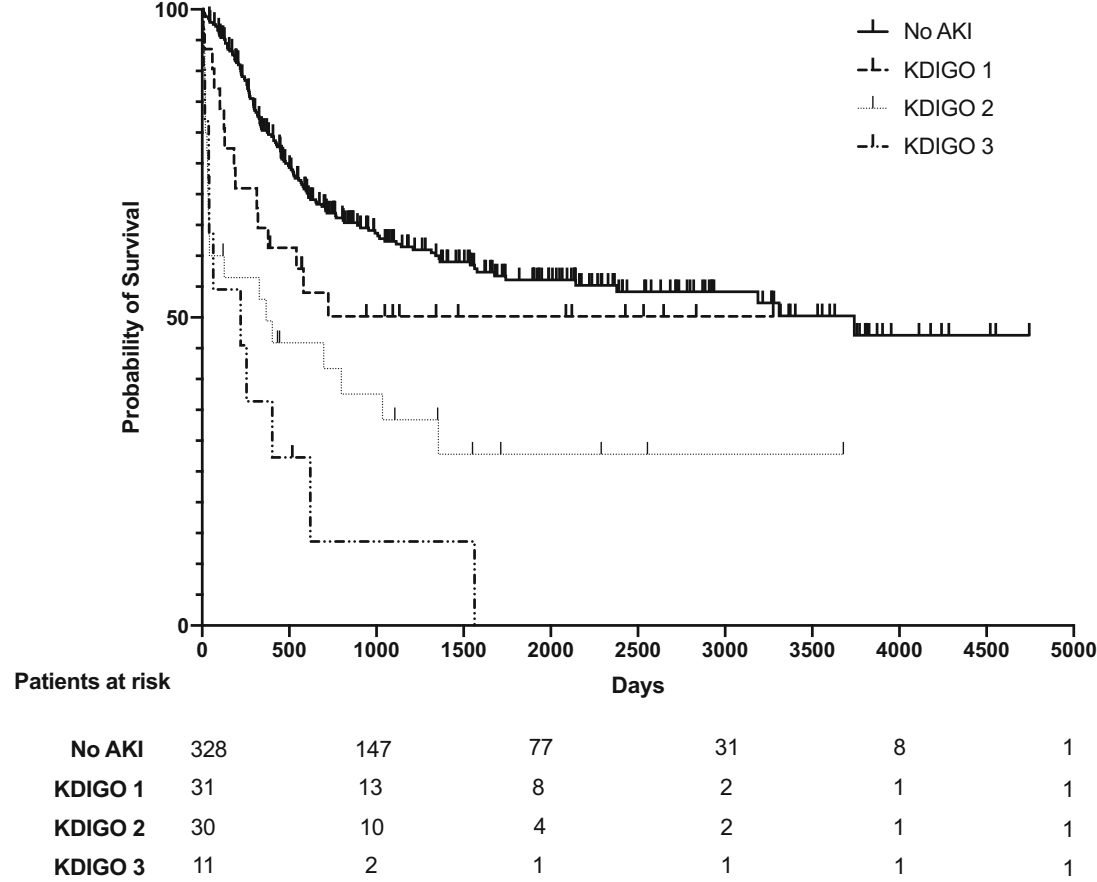

receive curative treatment. $\mathrm{SCr}$ values on day 90 after initiation of induction chemotherapy and at discharge from hospital remained higher in AML patients with AKI. Thus, it seems as if AKI in AML patients undergoing induction chemotherapy leads to a permanent restriction of kidney function, thereby adversely affecting the further clinical course of these patients after induction chemotherapy.

One of our key objectives was identifying risk factors associated with AKI in AML patients undergoing induction chemotherapy. Use of vancomycin and amphotericin B lipid formulation, diuretics, leukopenia, age, and hypoalbuminemia was already described as risk factors for AKI [8]. In our analysis, we confirmed the association of age with AKI in AML patients undergoing induction chemotherapy. We also found that lower albumin levels on the day of admission to hospital and especially throughout the clinical course of induction chemotherapy were associated with AKI. Decreased serum albumin levels are described to be a biomarker of poor prognosis including risk for AKI and mortality in patients treated on ICU [21-23]. Hypoalbuminemia has been described to be a predictor of mortality in different clinical setups [21, 22]. So far, albumin replacement studies in patients with sepsis and/or AKI did not improve outcomes, although the studied populations did not aim at hematological patients [24]. Prospective studies are needed to analyze whether prevention of hypoalbuminemia (e.g., by optimizing nutrition or replacing albumin) can minimize the risk for AKI in AML patients undergoing induction chemotherapy.

Potentially, nephrotoxic medications were documented for all patients and correlated with the incidence of AKI. We were

Table 7 Univariate and multivariate analysis associated with survival in AML patients

\begin{tabular}{|c|c|c|c|c|c|c|}
\hline \multirow{2}{*}{$\begin{array}{l}\text { Parameter } \\
\text { Age }>60\end{array}$} & \multicolumn{3}{|c|}{ Univariate analysis } & \multicolumn{3}{|c|}{ Multivariate analysis } \\
\hline & 2.309 & $1.708-3.121$ & $<0.001$ & 1.687 & $1.199-2.373$ & 0.003 \\
\hline Male sex & 1.348 & $1.002-1.814$ & 0.048 & & & \\
\hline Adverse-risk AML & 1.713 & $1.209-2.427$ & 0.002 & 1.984 & $1.357-2.902$ & $<0.001$ \\
\hline$>5$ days with fever & 1.216 & $0.902-1.640$ & 0.200 & & & \\
\hline Treatment on intensive care unit & 2.825 & $2.005-3.982$ & $<0.001$ & 1.644 & $1.087-2.485$ & 0.018 \\
\hline Complete remission after induction chemotherapy & 0.318 & $0.233-0.434$ & $<0.001$ & 0.415 & $0.297-0.581$ & $<0.001$ \\
\hline Stem cell transplantation as consolidation therapy & 0.394 & $0.292-0.533$ & $<0.001$ & 0.475 & $0.336-0.670$ & $<0.001$ \\
\hline Acute kidney injury during induction chemotherapy & 2.301 & $1.637-3.233$ & $<0.001$ & 1.761 & $1.184-2.619$ & 0.005 \\
\hline Acute kidney injury stage 1 or 2 during induction chemotherapy & 1.988 & $1.368-2.890$ & $<0.001$ & 1.606 & $1.051-2.456$ & 0.029 \\
\hline
\end{tabular}


able to confirm the finding of the study by Lahoti et al. regarding liposomal amphotericin $\mathrm{B}$ as an independent risk factor for AKI in AML patients undergoing induction chemotherapy. Furthermore, we observed a positive correlation of exposure to ACE inhibitors and AKI in AML patients undergoing induction chemotherapy. On the one hand, ACE inhibitors can cause prerenal AKI when afferent blood supply is compromised, which is present in sepsis as a result of disruption of renal perfusion [25]. Therefore, it would be of interest whether discontinuation and exchange of therapy with ACE inhibitors (e.g., by calcium channel blockers) in AML patients who are at risk of suffering from sepsis is recommendable. On the other hand, most of the AML patients taking ACE inhibitors probably have hypertensive kidney disease to some extent, thereby increasing risk for development of AKI from the beginning. The use of ACE inhibitors in prior studies was not associated with a high risk of death and was shown to be a protective factor in critically ill patients with acute renal failure [26]. Due to the retrospective nature of this study, we are not able to draw a final conclusion from this interesting finding.

In our study, we confirmed the KDIGO classifications as a prognostic model in AML patients undergoing induction chemotherapy. We were able to demonstrate a stepwise increase of mortality with increasing AKI severity. By using the KIDGO criteria, we pointed out that a relatively mild elevation in creatinine as small as $0.3 \mathrm{mg} / \mathrm{dl}$ (which might be missed in clinical practice) also adversely impacts the clinical course of these patients. We therefore advocate a sensitization not only for AKI stage 3, but for all AKI stages as we identified AKI stage 1 and 2 alone to be an independent risk factor for OS in AML patients undergoing induction chemotherapy.

In conclusion, the KIDGO classification for AKI is of high utility for mortality risk stratification in AML patients undergoing induction chemotherapy. We advocate a higher awareness of AKI, especially for mild AKI episodes which might often be overlooked. We further advise the accurate documentation of AKI episodes and the identification of patients who are prone and susceptible to AKI episodes (age, comorbidities, medication). We recommend incorporating that in the decision-making among nutrition, fluid management, the choice of antibiotic, and antifungal agents aim at decreasing the incidence of AKI. Furthermore, the early involvement of a nephrologist in patients at high risk for AKI may be reasonable for discussion of fluid management and applied medication. As to our limitations: The retrospective nature of our study limits the conclusions that can be drawn from the exhibited results. Second, our analysis did not include urine output in patients according to KDIGO criteria to classify AKI, which may have underestimated the incidence of AKI. The consideration of urine output and proteinuria is not often taken into consideration throughout AML induction therapy and should be considered as a screening and monitoring.
Prospective studies would be needed to determine whether the recommended strategies will help decrease the incidence of AKI in AML patients undergoing induction chemotherapy.

Supplementary Information The online version contains supplementary material available at https://doi.org/10.1007/s00277-021-04482-3.

Author contributions Olivier Ballo, Fagr Eladly, Fabian Finkelmeier, and Christian Brandts: conceptualization, data curation, formal analysis, investigation, methodology, writing - original draft. Stefan Büttner, Jan Alexander Stratmann, Sarah Rudolf, Uta Brunnberg, Björn Steffen, Sebastian Wagner, and Hubert Serve: review and editing.

Funding Open Access funding enabled and organized by Projekt DEAL.

\section{Declarations}

Conflict of interest The authors declare no conflicts of interest.

Open Access This article is licensed under a Creative Commons Attribution 4.0 International License, which permits use, sharing, adaptation, distribution and reproduction in any medium or format, as long as you give appropriate credit to the original author(s) and the source, provide a link to the Creative Commons licence, and indicate if changes were made. The images or other third party material in this article are included in the article's Creative Commons licence, unless indicated otherwise in a credit line to the material. If material is not included in the article's Creative Commons licence and your intended use is not permitted by statutory regulation or exceeds the permitted use, you will need to obtain permission directly from the copyright holder. To view a copy of this licence, visit http://creativecommons.org/licenses/by/4.0/.

\section{References}

1. Pemmaraju N, Kantarjian H, Garcia-Manero G, Pierce S, CardenasTuranzas M, Cortes J, Ravandi F (2015) Improving outcomes for patients with acute myeloid leukemia in first relapse: a single center experience. Am J Hematol 90(1):27-30. https://doi.org/10.1002/ ajh. 23858

2. Tiong IS, Reynolds J, Bradstock KF, Seymour JF, Wei AH, Australasian L, Lymphoma G (2018) Dissecting causes for improved survival among patients with acute myeloid leukemia in two different eras receiving identical regimens in sequential randomized studies. Blood Cancer J 8(9):84. https://doi.org/10.1038/ s41408-018-0121-4

3. Othus M, Kantarjian H, Petersdorf S, Ravandi F, Godwin J, Cortes J, Pierce S, Erba H, Faderl S, Appelbaum FR, Estey E (2014) Declining rates of treatment-related mortality in patients with newly diagnosed AML given 'intense' induction regimens: a report from SWOG and MD Anderson. Leukemia 28(2):289-292. https://doi. org/10.1038/leu.2013.176

4. Buckley SA, Othus M, Estey EH, Walter RB (2015) The treatmentrelated mortality score is associated with non-fatal adverse events following intensive AML induction chemotherapy. Blood Cancer J 5:e276. https://doi.org/10.1038/bcj.2014.97

5. Ronco C, Bellomo R, Kellum JA (2019) Acute kidney injury. Lancet 394(10212):1949-1964. https://doi.org/10.1016/S01406736(19)32563-2

6. Wiersema R, Eck RJ, Haapio M, Koeze J, Poukkanen M, Keus F, van der Horst ICC, Pettila V, Vaara ST (2019) Burden of acute 
kidney injury and 90-day mortality in critically ill patients. BMC Nephrol 21(1):1. https://doi.org/10.1186/s12882-019-1645-y

7. Du Plessis L, Rassekh SR, Mammen C (2018) High incidence of acute kidney injury during chemotherapy for childhood acute myeloid leukemia. Pediatr Blood Cancer 65(4). https://doi.org/10. 1002/pbc. 26915

8. Lahoti A, Kantarjian H, Salahudeen AK, Ravandi F, Cortes JE, Faderl S, O’Brien S, Wierda W, Mattiuzzi GN (2010) Predictors and outcome of acute kidney injury in patients with acute myelogenous leukemia or high-risk myelodysplastic syndrome. Cancer 116(17):4063-4068. https://doi.org/10.1002/cncr.25306

9. Wiernik PH, Case DC Jr, Periman PO, Arlin ZA, Weitberg AB, Ritch PS, Todd MB (1989) A multicenter trial of cytarabine plus idarubicin or daunorubicin as induction therapy for adult nonlymphocytic leukemia. Semin Oncol 16(1 Suppl 2):25-29

10. Hiddemann W, Kreutzmann H, Straif K, Ludwig WD, Mertelsmann R, Donhuijsen-Ant R, Lengfelder E, Arlin Z, Buchner T (1987) High-dose cytosine arabinoside and mitoxantrone: a highly effective regimen in refractory acute myeloid leukemia. Blood 69(3):744-749

11. Krug U, Buchner T, Berdel WE, Muller-Tidow C (2011) The treatment of elderly patients with acute myeloid leukemia. Dtsch Arztebl Int 108(51-52):863-870. https://doi.org/10.3238/arztebl. 2011.0863

12. Dohner H, Estey EH, Amadori S, Appelbaum FR, Buchner T, Burnett AK, Dombret H, Fenaux P, Grimwade D, Larson RA, Lo-Coco F, Naoe T, Niederwieser D, Ossenkoppele GJ, Sanz MA, Sierra J, Tallman MS, Lowenberg B, Bloomfield CD, European L (2010) Diagnosis and management of acute myeloid leukemia in adults: recommendations from an international expert panel, on behalf of the European LeukemiaNet. Blood 115(3):453474. https://doi.org/10.1182/blood-2009-07-235358

13. Neumann S, Krause SW, Maschmeyer G, Schiel X, von LilienfeldToal M, Infectious Diseases Working P, German Society of H, Oncology (2013) Primary prophylaxis of bacterial infections and Pneumocystis jirovecii pneumonia in patients with hematological malignancies and solid tumors : guidelines of the Infectious Diseases Working Party (AGIHO) of the German Society of Hematology and Oncology (DGHO). Ann Hematol 92(4):433442. https://doi.org/10.1007/s00277-013-1698-0

14. Mellinghoff SC, Panse J, Alakel N, Behre G, Buchheidt D, Christopeit M, Hasenkamp J, Kiehl M, Koldehoff M, Krause SW, Lehners N, von Lilienfeld-Toal M, Lohnert AY, Maschmeyer G, Teschner D, Ullmann AJ, Penack O, Ruhnke M, Mayer K, Ostermann H, Wolf HH, Cornely OA (2018) Primary prophylaxis of invasive fungal infections in patients with haematological malignancies: 2017 update of the recommendations of the Infectious Diseases Working Party (AGIHO) of the German Society for Haematology and Medical Oncology (DGHO). Ann Hematol 97(2):197-207. https://doi.org/10.1007/s00277-0173196-2

15. Freifeld AG, Bow EJ, Sepkowitz KA, Boeckh MJ, Ito JI, Mullen CA, Raad RKV II, Young JA, Wingard JR, Infectious Diseases Society of A (2011) Clinical practice guideline for the use of antimicrobial agents in neutropenic patients with cancer: 2010 update by the infectious diseases society of America. Clin Infect Dis 52(4): e56-e93. https://doi.org/10.1093/cid/cir073

16. Swerdlow SH, Campo E, Pileri SA, Harris NL, Stein H, Siebert R, Advani R, Ghielmini M, Salles GA, Zelenetz AD, Jaffe ES (2016) The 2016 revision of the World Health Organization classification of lymphoid neoplasms. Blood 127(20):2375-2390. https://doi.org/ 10.1182/blood-2016-01-643569

17. Fitzgerald JC, Basu RK, Akcan-Arikan A, Izquierdo LM, Pineres Olave BE, Hassinger AB, Szczepanska M, Deep A, Williams D, Sapru A, Roy JA, Nadkarni VM, Thomas NJ, Weiss SL, Furth S, Sepsis Prevalence O, Therapies Study I, Pediatric Acute Lung I, Sepsis Investigators N (2016) Acute kidney injury in pediatric severe sepsis: an independent risk factor for death and new disability. Crit Care Med 44(12):2241-2250. https://doi.org/10.1097/CCM. 0000000000002007

18. Petri CR, O'Donnell PH, Cao H, Artz AS, Stock W, Wickrema A, Hard M, van Besien K (2014) Clofarabine-associated acute kidney injury in patients undergoing hematopoietic stem cell transplant. Leuk Lymphoma 55(12):2866-2873. https://doi.org/10.3109/ 10428194.2014.897701

19. Darmon M, Vincent F, Canet E, Mokart D, Pene F, Kouatchet A, Mayaux J, Nyunga M, Bruneel F, Rabbat A, Lebert C, Perez P, Renault A, Meert AP, Benoit D, Hamidfar R, Jourdain M, Schlemmer B, Chevret S, Lemiale V, Azoulay E (2015) Acute kidney injury in critically ill patients with haematological malignancies: results of a multicentre cohort study from the Groupe de Recherche en Reanimation Respiratoire en Onco-Hematologie. Nephrol Dial Transplant 30(12):2006-2013. https://doi.org/10. 1093/ndt/gfv372

20. Peerapornratana S, Manrique-Caballero CL, Gomez H, Kellum JA (2019) Acute kidney injury from sepsis: current concepts, epidemiology, pathophysiology, prevention and treatment. Kidney Int 96(5):1083-1099. https://doi.org/10.1016/j.kint.2019.05.026

21. Aman J, van der Heijden M, van Lingen A, Girbes AR, van Nieuw Amerongen GP, van Hinsbergh VW, Groeneveld AB (2011) Plasma protein levels are markers of pulmonary vascular permeability and degree of lung injury in critically ill patients with or at risk for acute lung injury/acute respiratory distress syndrome. Crit Care Med 39(1):89-97. https://doi.org/10.1097/CCM. 0b013e3181feb46a

22. Novak F, Borovska J, Vecka M, Rychlikova J, Vavrova L, Petraskova H, Zak A, Novakova O (2017) Plasma phospholipid fatty acid profile is altered in both septic and non-septic critically ill: a correlation with inflammatory markers and albumin. Lipids 52(3):245-254. https://doi.org/10.1007/s11745-016-4226-x

23. Schmidt de Oliveira-Netto AC, Morello LG, Dalla-Costa LM, Petterle RR, Fontana RM, Conte D, Pereira LA, Raboni SM (2019) Procalcitonin, C-reactive protein, albumin, and blood cultures as early markers of sepsis diagnosis or predictors of outcome: a prospective analysis. Clin Pathol 12:2632010X19847673. https:// doi.org/10.1177/2632010X19847673

24. Takegawa R, Kabata D, Shimizu K, Hisano S, Ogura H, Shintani A, Shimazu T (2019) Serum albumin as a risk factor for death in patients with prolonged sepsis: an observational study. J Crit Care 51:139-144. https://doi.org/10.1016/j.jcrc.2019.02.004

25. Textor SC (1997) Renal failure related to angiotensin-converting enzyme inhibitors. Semin Nephrol 17(1):67-76

26. Silva Junior GB, Daher Ede F, Mota RM, Menezes FA (2006) Risk factors for death among critically ill patients with acute renal failure. Sao Paulo Med J 124(5):257-263. https://doi.org/10.1590/s151631802006000500004

Publisher's note Springer Nature remains neutral with regard to jurisdictional claims in published maps and institutional affiliations. 